\title{
Epigenetically deregulated miR-200c is involved in a negative feedback loop with DNMT3a in gastric cancer cells
}

\author{
YINGFEI LI ${ }^{1}$, YUQIANG NIE ${ }^{1}$, SANFANG TU ${ }^{3}$, HONG WANG $^{1}$, YONGJIAN ZHOU ${ }^{1}$, \\ YANLEI DU ${ }^{1}$, JIE $\mathrm{CAO}^{2}$ and MIN $\mathrm{YE}^{1}$ \\ Departments of ${ }^{1}$ Gastroenterology and Hepatology, and ${ }^{2}$ General Surgery, Guangzhou Digestive Diseases Center, \\ Guangzhou First People's Hospital, Guangzhou Medical University, Guangzhou, Guangdong 510180; \\ ${ }^{3}$ Department of Hematology, Zhujiang Hospital, Southern Medical University, Guangzhou, Guangdong 510280, P.R. China
}

Received February 17, 2016; Accepted March 28, 2016

DOI: 10.3892/or.2016.4996

\begin{abstract}
Aberrant methylation of miRNAs is commonly observed in cancers. In the present study, we investigated the regulation of the miR-200 family and its role in regulating DNA methylation events in gastric cancer (GC). We demonstrated that miR-200c was aberrantly expressed in GC and associated with histologic type and tumor progression. Hypermethylation of the promoter region was found to be responsible for the loss of miR-200c in GC cells. Demethylation agents led to recovery of miR-200c expression in GC cell lines. Moreover, DNMT3a knockdown abolished the hypermethylation of the miR-200c gene and induced upregulation of miR-200c expression, whereas ectopic DNMT3a expression increased miR-200c promoter methylation and decreased miR-200c expression. Conversely, transfection of miR-200c led to downregulation of DNMT3a protein and induced endogenous pre-miR-200c and pri-miR-200c re-expression. Luciferase assays confirmed miR-200c binding to the DNMT3a 3'UTR. Finally, ectopic expression of miR-200c or knockdown of DNMT3a expression impeded GC cell growth, migration and invasion. Taken together, these observations demonstrates a novel epigenetic feedback loop between miR-200c and DNMT3a in the carcinogenesis and progression of GC.
\end{abstract}

\section{Introduction}

Gastric cancer (GC) is one of the most common malignant tumors in China with a high incidence and mortality rate (1).

Correspondence to: Professor Yuqiang Nie, Department of Gastroenterology and Hepatology, Guangzhou Digestive Diseases Center, Guangzhou First People's Hospital, Guangzhou Medical University, Guangzhou, Guangdong 510180, P.R. China

E-mail: nieyq@medmail.com.cn

Abbreviations: miRNAs, microRNAs; GC, gastric cancer; DNMT3a, DNA (cytosine-5-)-methyltransferase $3 \alpha$; EMT, epithelialmesenchymal transition;

Key words: gastric cancer, hsa-miR-200c, DNMT3a, methylation, feedback loop
Local invasion and distant metastasis are leading causes for the dismal outcome of GC patients. Despite recent advances in surgical techniques and new chemotherapy regimens, the 5-year survival rate of GC patients remains low. Therefore, investigation of molecular mechanisms underlying GC development and progression are urgently needed to develop novel diagnostics and therapies for GC.

Aberrant expression of microRNAs (miRNAs) is assumed to play an important role in cancer development by regulating cell proliferation, differentiation, apoptosis and carcinogenesis (2). Previous studies have shown that many miRNAs are aberrantly expressed during GC progression $(3,4)$. Although the mechanisms underlying miRNA dysregulation in GC remain a challenge, recent studies have shown that promoter $\mathrm{CpG}$ island hypermethylation plays a key role in the silencing of numerous miRNAs (5). For example, DNA methylation of miR-210 increases the proliferation of gastric epithelium during chronic $H$. pylori infection (6). Moreover, miR-10b functions as a tumor suppressor but its hypermethylation occurs in GC (7). Thus, aberrant hypermethylation events in the regulatory regions of miRNAs may play a key role in cancer development. In contrast, miRNAs can also modulate epigenetic regulatory mechanisms of gene expression by targeting DNA methyltransferase enzymes (DNMT1, DNMT3a and DNMT3b). For example, the miR-29 family directly targets DNMT3a and DNMT3b in lung cancer, thereby protecting against changes in the existing DNA methylation status and suppressing tumorigenesis (8). Moreover, miR-152 can negatively regulate DNMT1 directly by targeting the 3'UTR of DNMT1 in breast (9), ovarian (10) and liver cancer (11), respectively. These findings indicate an important role for miRNAs which target DNMTs during carcinogenesis. Collectively, the epigenetic silencing of both the miRNAs and miRNAs targeting DNMTs have been described to play a role in cancer.

To further explore the underlying molecular mechanisms of this interaction in GC, we hypothesized that an interaction between miRNA and DNMTs could be contributing to gastric carcinogenesis. In a previous study, the miR-200 family was proved to function as a tumor suppressor by suppressing other genes (12), but frequently silenced by hypermethylation in many types of cancers (13-16). Intriguingly, the miR-200 
family was predicted to target DNMT3a directly, as predicted by several in silico methods for target gene prediction (PicTAR, TargetScan and MiRanda). Given the fact that the methylationsensitive miRNAs and DNMT-targeting miRNAs may have the ability to form an epigenetic-miRNA regulatory circuit, we hypothesized that a negative feedback loop exists between the miR-200 family and DNMT3a in GC.

To test this hypothesis, we specifically investigated: i) the expression of miR-200 family and DNM3a in a panel of GC tissues; ii) the probable associations between miR-200 family levels and the clinicopathological features of patients with GC; iii) the probable negative feedback loop between the miR-200 family and DNA methyltransferase; and iv) the probable roles of miR-200c and DNMT3a in cell proliferation and invasion. The answer to these questions would provide new insights into the molecular mechanisms involved in GC development and metastasis as well as provide a new strategy for GC diagnostic and treatment.

\section{Materials and methods}

Cell culture, 5-Aza-dC treatment and transfection. The human GC cell lines NCI-N87 (well-differentiated), SGC7901 and AGS (moderately differentiated), HGC27 and MGC803 (poorly differentiated), GES-1 (the human normal gastric cell line) were cultured in RPMI-1640 medium (HyClone, Logan, UT, USA) supplemented with $10 \%$ fetal calf serum and incubated at $37^{\circ} \mathrm{C}$ with $5 \% \mathrm{CO}_{2}$ in a humidified atmosphere. To examine the role of methylation in the regulation of miRNA expression, GC cells at $5 \times 10^{5}$ cells $/ \mathrm{ml}$ were cultured with 5-aza-2'-deoxycytidine (5-Aza-dC) (Sigma-Aldrich, St. Louis, $\mathrm{MO}, \mathrm{USA})$ at $2.5 \mu \mathrm{M}$ for $72 \mathrm{~h}$. Transfections were performed using a Lipofectamine 2000 kit (Invitrogen, Carlsbad, CA, USA) according to the manufacturer's instructions. A total of $100 \mathrm{nM}$ of siRNA against DNMT3a (sc-37757; Santa Cruz Biotechnology, Santa Cruz, CA, USA), double-stranded miR-200c mimics (50 nM), single-stranded miR-200c inhibitor (100 nM) (RiboBio, Guangzhou, China) or their relative negative control RNAs were introduced into cells.

Primary tissue samples. Cancer and adjacent non-cancerous tissues were obtained from 46 GC patients who underwent gastrectomy between January 2005 and December 2012 at Guangzhou First People's Hospital. Tissue samples were immediately embedded in liquid nitrogen and then stored at $-80^{\circ} \mathrm{C}$ until RNA, DNA and protein could be extracted. Written consent was obtained from each patient before operation. The study protocol was approved by the Medical Ethics Committee of Guangzhou First People's Hospital.

Plasmids and luciferase activity assays. For DNMT3a overexpression, the coding sequence of DNMT3a was PCR-amplified $(2,739 \mathrm{bp})$ and cloned into pcDNA3.1 ${ }^{+}$. The primers used were as follows: DNMT3a sense, 5'-cgcggatccgecaccATGCCCGCCATGCCCTCCAG-3' and antisense, 5'-ataagaatgcggecgc TTACACACACGCAAAATACTCCTT-3'. Insertion was confirmed by Sanger sequencing. Expression plasmids were transfected into MGC803 cells using Lipofectamine 2000 according to the manufacturer's instructions. For luciferase reporter assays, the 3'UTRs of DNMT3a containing an intact
miR-200c recognition sequence was PCR-amplified (1,303 bp) and cloned into psiCHECK ${ }^{\mathrm{TM}}-2$ (Promega, Madison, WI, USA). A psiCHECK ${ }^{\mathrm{TM}}-2$ construct containing the DNMT3a 3'UTR with mutations in the seed sequence was synthesized using a QuikChange Site-Directed Mutagenesis kit (Agilent Technologies, Santa Clara, CA, USA). All constructs were confirmed by Sanger sequencing. The primers used were as follows: DNMT3a-wild-type sense, 5'-CCGCTCGAGGGG ACATGGGGGCAAACTG-3' and antisense, 5'-CATAAGA ATGCGGCCGCTACGTTTTGTATGTTTTTTTATTTG-3'; mutant sense, 5'-CCACACAAGACATTTTTCTAGTCATA ATCAGGTGCCTACCACACAGG and antisense, 5'-CCT GTGTGGTAGGCACCTGATTATGACTAGAAAAATGTC TTGTGTGG-3'. SGC7901 cells at 50\% confluency were transiently transfected with $0.5 \mu \mathrm{g}$ of reporter plasmids alone or co-transfected with or without miRNA mimics or inhibitors using Lipofectamine 2000 according to the protocol. The luciferase activities of both firefly and Renilla luciferase were quantified by a Dual-Luciferase Reporter Assay System and the relative luciferase activity was obtained according to the manufacturer's instructions (GloMax Detection System) (both from Promega). Independent triplicate experiments were carried out for all samples.

RNA extraction and quantitative real-time PCR. RNA was isolated using TRIzol (Invitrogen) according to the manufacturer's protocol. The expression levels of miR-200b, miR-200c and $\mathrm{miR}-141$ were measured by reverse transcription-PCR analysis according to the miRCURY LNA ${ }^{\mathrm{TM}}$ Universal RT microRNA PCR manual (Exiqon, Vedbaek, Denmark). The expression of miR-200 family member was normalized to that of RNU6B, small nuclear RNA expression.

DNA methylation analysis. The CpG island of the miR-200c gene was searched in the miRBase database (release 24 June, 2013), the UCSC Genome Browser on Human Assembly (hg 19; release February 2009) and PubMed (17). Genomic DNA from patient samples and MGC803 cells were isolated by QIAamp DNA Mini kit (Qiagen, Germantown, MD, USA). Bisulfite conversion of genomic DNA was performed using the EpiTect Bisulfite kit (Qiagen) and then amplified by PCR using methylation-specific primers. The primers used were as follows: miR-200c sense, 5'-GGGGTAGGGGAAGGTGGT TTA-3' and antisense, 5'-CACCACCCCAATCCCTAAAAAC ACT-3'; the primer used for sequencing was as follows: GGGAAGGTGGTTTAGA. We investigated the methylation status of GC tissues using pyrosequencing. DNA methylation analysis of bisulfite PCR amplicons for all samples was performed on the PyroMark Q24 instrument according to the manufacturer's instructions (Qiagen). DNA methylation level was defined as the average percent methylation of each individual $\mathrm{CpG}$-unit averaged across the regions. We evaluated the impact of DNMT3a overexpression or invalidation on the methylation status of the miR-200c gene using bisulfite sequencing PCR. The primers used for bisulfite sequencing PCR were as follows: BSP-miR-200c sense, 5'-TTTTAG TATTTATTTTTTGGGGGTAG-3' and antisense, 5'-CAC CTTAAATCAAACAACTTCAAAC-3'. The products of interest (375 bp) were purified by the DNA Gel Extraction kit, ligated into the Pmd19-T vector (Takara, Dalian, China) and 
transformed into Escherichia coli DH5a competent cells. Insertion was confirmed by restriction enzyme digestion, and 10 clones were chosen for sequencing.

Immunohistochemistry. For immunohistochemistry, paraffin sections were deparaffinized and incubated with a primary rabbit polyclonal DNMT3a antibody (Santa Cruz Biotechnology) at a dilution of 1:150 in a $4^{\circ} \mathrm{C}$ moist chamber overnight. Immunohistochemistry was independently evaluated by two researchers who were blinded to the patient outcome. The evaluation was based on the staining intensity. Staining intensity for DNMT3a was scored as 0 (negative); 1 (weak); 2 (moderate); and 3 (strong). The specimens were divided into two groups according to their scores: 0 and 1 were considered as the low expression group; 2 and 3 were considered as the high expression group. In the event of a discrepancy in scoring, the slides were re-examined by both pathologists under a microscope.

Western blotting. To isolate the proteins, cells collected by 6-well plates were washed once in phosphate-buffered saline and lysed in lysis buffer. Each protein sample $(15 \mu \mathrm{g})$ was resolved by sodium dodecylsulfate-polyacrylamide gel electrophoresis, transferred onto a polyvinylidene difluoride membrane, and incubated with a monoclonal antibody against DNMT3a $(1: 1,000)$ (Santa Cruz Biotechnology) or GAPDH $(1: 10,000)$ (KangCheng Bio-tech, Shanghai, China). The signals were detected by incubation with secondary antibodies $(1: 20,000$ for goat anti-rabbit IgG, and 1:10,000 for rabbit anti-mouse IgG; Southern Biotech) labeled with the ECL detection system.

Cell proliferation, invasion and wound-healing assays. Cell proliferation assays were performed using Cell Counting Kit-8 (Beyotime, Hangzhou, China) after transfection of the miR-200c mimics or DNMT3a siRNA. Absorbance was measured at $450 \mathrm{~nm}$ using a microplate reader. Cell invasion assays were assessed at $48 \mathrm{~h}$ after transfection using Matrigel Invasion Chambers (BD Biosciences, Franklin Lakes, NJ, USA). For the wound-healing assays, cell monolayers transfected with miR-200c mimics or DNMT3a siRNA were scratched with a clean pipette tip, and cell migration was observed for up to $48 \mathrm{~h}$.

Statistical analysis. All experiments were conducted in triplicate. Differences between groups (continuous variables) were analyzed by the Student's t-test. The relationship between the expression of miR-200c and clinicopathological factors was analyzed by Chi-square tests. All statistical analyses were twosided and were performed using SPSS software (version 16.0; SPSS, Inc., Chicago, IL, USA), and $\mathrm{P}<0.05$ was considered to indicate a statistically significant result.

\section{Results}

miR-200c is downregulated in GC tissues. The miR-200 family can be grouped into two subfamilies in two ways. The first way is functionally, according to the presence of two types of seed sequences: miR-200a/miR-141 with a common sequence of AAcACUG, and miR-200b/miR-200c/miR-429 with AAuACUG. The second way is genetically, according
Table I. miR-200c expression and clinicopathological characteristics of the gastric cancer cases.

\begin{tabular}{|c|c|c|c|}
\hline Characteristic & $\begin{array}{l}\text { High expression } \\
\qquad(n=23)\end{array}$ & $\begin{array}{l}\text { Low expression } \\
\quad(\mathrm{n}=23)\end{array}$ & P-value \\
\hline $\begin{array}{l}\text { Age, years } \\
(\text { mean } \pm \text { SD })\end{array}$ & $59.0 \pm 12.3$ & $60.0 \pm 6.9$ & 0.725 \\
\hline Gender & & & 0.760 \\
\hline Male & 15 & 14 & \\
\hline Female & 8 & 9 & \\
\hline Histological type & & & $0.018^{\mathrm{a}}$ \\
\hline Intestinal & 16 & 8 & \\
\hline Diffuse & 7 & 15 & \\
\hline Depth of invasion & & & $0.010^{\mathrm{a}}$ \\
\hline $\mathrm{T} 1+\mathrm{T} 2$ & 11 & 3 & \\
\hline $\mathrm{T} 3+\mathrm{T} 4$ & 12 & 20 & \\
\hline $\begin{array}{l}\text { Lymph node } \\
\text { metastasis }\end{array}$ & & & 0.063 \\
\hline Negative (N0) & 11 & 5 & \\
\hline Positive (N1-N3) & 12 & 18 & \\
\hline Organ metastasis & & & 0.710 \\
\hline Negative (N0) & 19 & 18 & \\
\hline Positive (N1-N3) & 4 & 5 & \\
\hline Stage & & & $0.038^{\mathrm{a}}$ \\
\hline I+II & 14 & 7 & \\
\hline III+IV & 9 & 16 & \\
\hline Tumor site & & & 0.710 \\
\hline Cardia cancer & 5 & 4 & \\
\hline Non-cardia cancer & 18 & 19 & \\
\hline
\end{tabular}

The tumor stage was determined based on the surgical and imaging findings according to the tumor-node-metastasis (TNM) staging system of the American Joint Committee on Cancer (AJCC). The histological diagnosis of each case was doubly confirmed by two experienced pathologists independently. The pathohistological classification was based on Lauren's system. $\left({ }^{\mathrm{a}} \mathrm{P}<0.05\right)$.

to the location in two gene clusters on two different chromosomes: $\mathrm{miR}-200 \mathrm{~b} / \mathrm{miR}-200 \mathrm{a} / \mathrm{miR}-429$ on chr. 1 , and $\mathrm{miR}-200 \mathrm{c} / \mathrm{miR}-141$ on chr. 12. In the present study, we performed most experiments with three miR-200 family members: miR-200b, miR-200c and miR-141, since they represent members of both subfamilies. As shown in Fig. 1A, we analyzed the expression levels of all three miR-200 family members in 46 pairs of GC and their corresponding non-tumor tissues. In qRT-PCR analysis, miR-200c was significantly downregulated in the GC tissues compared with that noted in the adjacent non-tumor tissues $(\mathrm{P}<0.05)$. There was no significant difference in miR-200b $(\mathrm{P}=0.495)$ and miR-141 expression levels $(\mathrm{P}=0.561)$ between the $\mathrm{GC}$ and non-tumor tissues. The high-expression group was defined as cases with miR-200c expression above the median value, while the remaining cases were included into the low-expression group. We further compared the clinicopathological parameters of GC according to their miR-200c expression level. As shown 
A
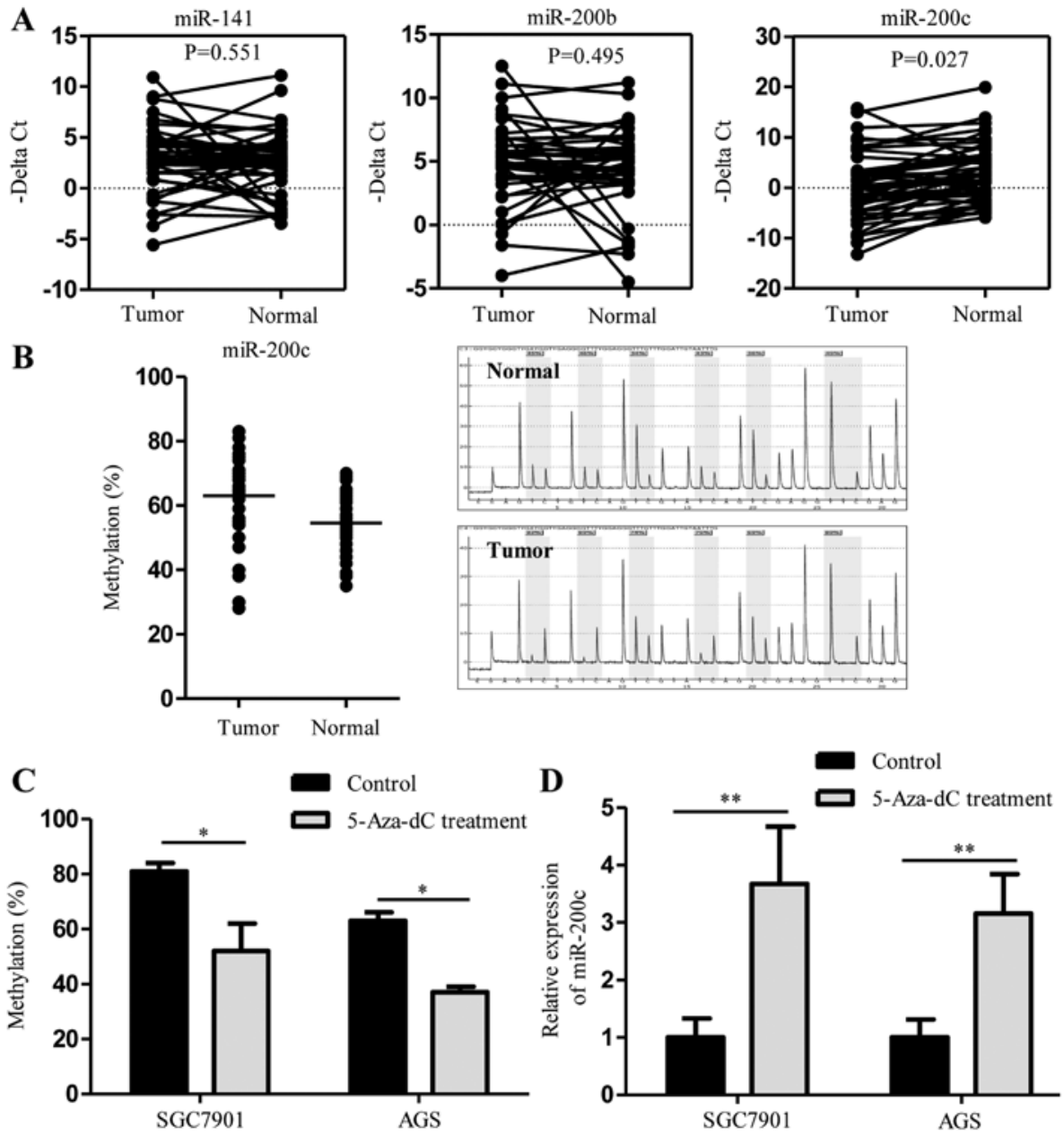

Figure 1. Expression levels of the miR-200 family and methylation analysis of miR-200c. (A) Tumor and non-tumor tissue pairs from $46 \mathrm{GC}$ patients were examined using reverse transcription-PCR method. The expression levels of candidates were normalized to U6 expression $(\Delta \mathrm{Ct})$. miR-200c, but not miR-200b or miR-141, was significantly downregulated in the GC tissues compared with the adjacent non-tumor tissues. (B) Methylation pyrosequencing analysis revealed that the miR-200c promoter region was significantly hypermethylated in the GC tissues compared with adjacent non-tumor tissues in 39 pairs of GC tissues. (C) Pyrosequencing analysis showed that treatment with 5-aza-dC decreased the methylation status of the miR-200c promoter region in the SGC7901 and AGS cells. (D) Reverse transcription-PCR analysis showed that treatment with 5-aza-dC resulted in the upregulation of miR-200c in the SGC7901 and AGS cells $\left({ }^{*} \mathrm{P}<0.05,{ }^{* *} \mathrm{P}<0.01\right)$.

in Table I, the diffuse histologic type, deeper invasion and advanced stage of $\mathrm{GC}$ were all associated with low expression of miR-200c.

Hypermethylation of the miR-200c CpG island promoter causes downregulation of $m i R-200 c$. To determine the methylation status of the promoter region in the miR-200c gene, we analyzed the methylation status at six $\mathrm{CpG}$ dinucleotide sites of the miR-200c locus (chr12:7072639-7072665) in 39 pairs of GC and matched adjacent non-tumor tissues. Pyrosequencing analysis revealed that the miR-200c promoter region was significantly hypermethylated in the GC tissues when compared with that in the adjacent non-tumor tissues (63.0\% for GC tissues vs. $54.6 \%$ for non-tumor tissues; $\mathrm{P}<0.01$; Fig. 1B). In addition, we treated GC cell lines with the demethylating agent, 5-aza-dC. Pyrosequencing analysis showed that treatment with 5-aza-dC decreased the methylation status of the miR-200c promoter region in the SGC7901 cells $(80.7 \%$ for untreated controls vs. $52.3 \%$ for 5 -aza-dC; $\mathrm{P}<0.05)$ and in the AGS cells $(63.0 \%$ for untreated control vs. $37.3 \%$ for 5 -aza-dC; $\mathrm{P}<0.05$ ) (Fig. 1C). Demethylation of the miR-200c promoter region resulted in the upregulation of miR-200c in the SGC7901 and AGS cells as assayed by qRT-PCR $(\mathrm{P}<0.01$ for all) (Fig. 1D). Together, these data indicate that DNA methylation may be involved in miR-200c expression.

DNMT3a upregulation is responsible for the hypermethylation of the miR-200c gene promoter. Previous studies have shown that DNMT3a overexpression contributes to gene promoter hypermethylation and is associated with the malignant potential and poor prognosis of human cancer. In the present study, we found that the expression of DNMT3a in five GC cell lines was higher than that in immortal gastric epithelial GES-1 cells (Fig. 2A). Consistent with the western blot results, the expression of DNMT3a was strongly increased in the GC tissues than that noted in the non-tumor tissues by immunohistochemistry. In GC, expression of DNMT3a was mainly observed in the nucleus. Weak staining was also observed in the cytoplasm (Fig. 2B). Among the 46 paired samples, DNMT3a high expression was found in 31/46 of the GC samples. This was significantly higher compared to that noted in the paired control samples $(67.4$ vs. $34.8 \%$; $\mathrm{P}<0.01)$. 
A

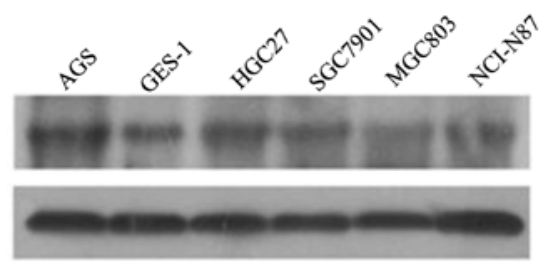

B

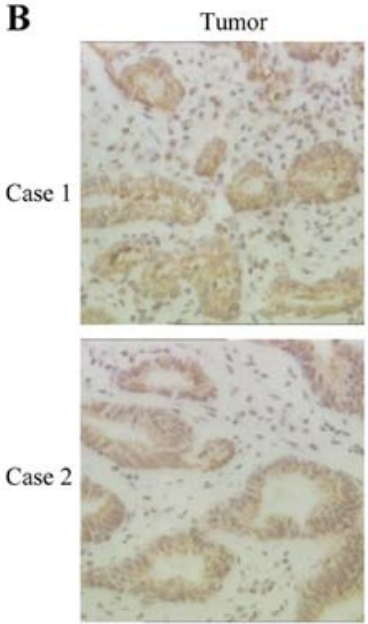

Normal

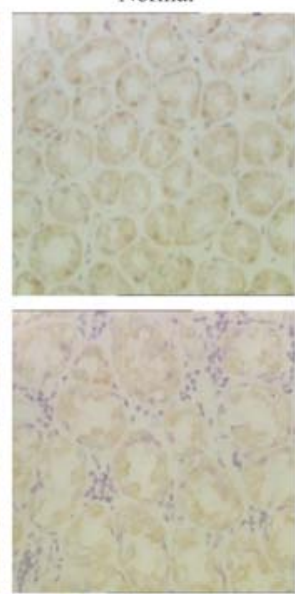

Figure 2. Differential expression of DNMT3a in cell lines and tissues. (A) Western blotting showed that the expression of DNMT3a in five GC cell lines was higher than that in immortal gastric epithelial GES-1 cells. (B) Immunohistochemistry assays showed that the expression of DNMT3a in GC tissues was significantly higher compared to that noted in the adjacent non-tumor tissues (magnification, $\mathrm{x} 200$ ).

A

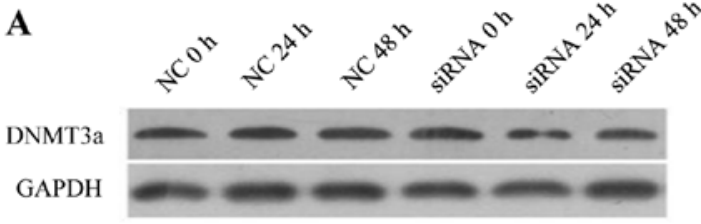

C miR-200e CpG island (Chr12: 7072470-7072844)
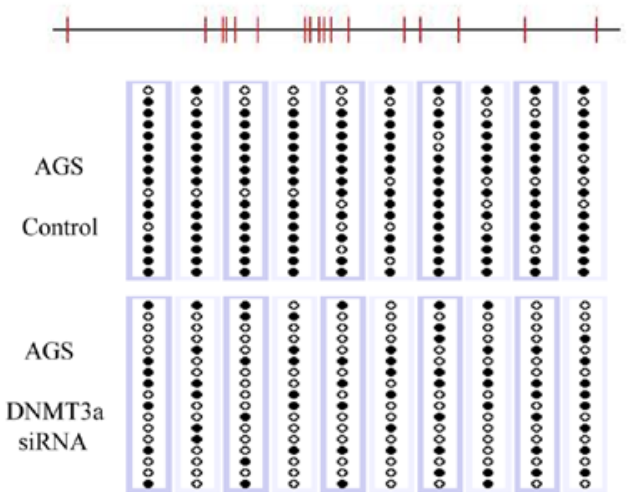

MGC803

Control
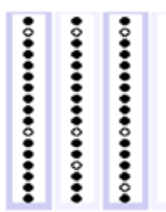

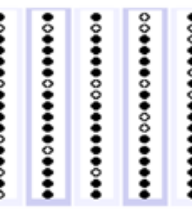

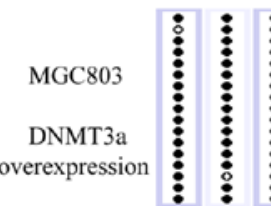

B

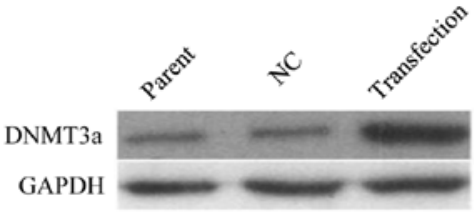

D
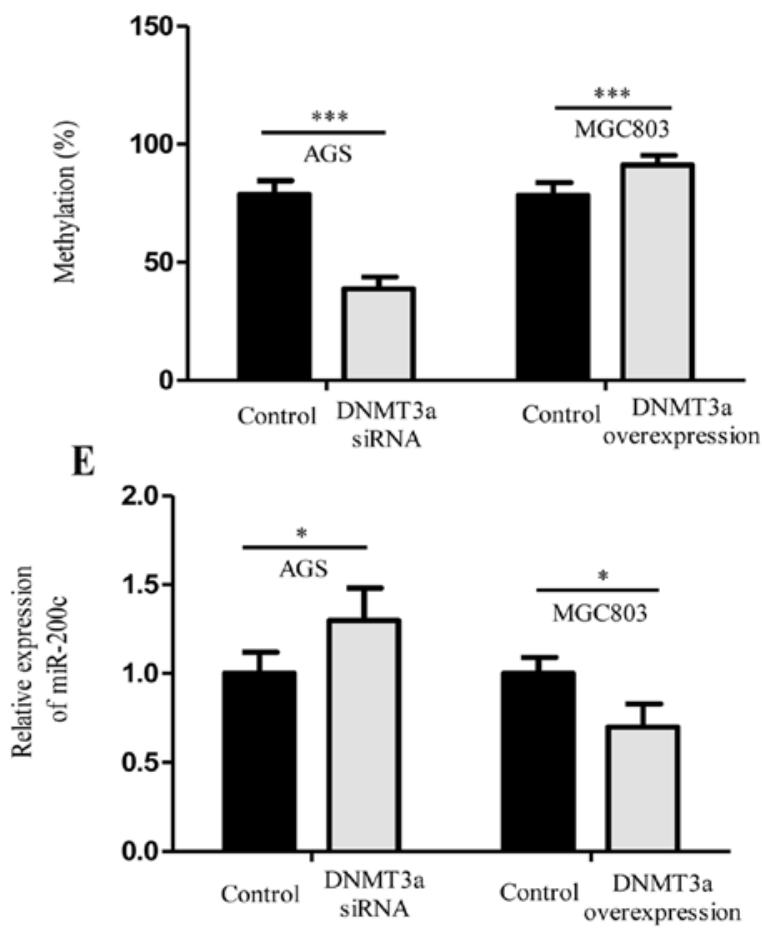

Figure 3. DNMT3a upregulation is responsible for the hypermethylation of the miR-200c gene promoter. (A) Knockdown of DNMT3a protein in AGS cells following 0,24 and $48 \mathrm{~h}$ of transfection with $100 \mathrm{nM}$ siRNA relative to negative controls. (B) Enhanced expression of DNMT3a protein in the MGC803 cells after stable transfection with the DNMT3a expression vector. (C and D) Bisulfite sequencing PCR showed that DNMT3a knockdown decreased the methylation status of the miR-200c promoter region in the AGS cells, and enhanced DNMT3a expression increased the methylation status of the miR-200c promoter region in the MGC803 cells. (E) Knockdown of DNMT3a resulted in upregulation of miR-200c expression, whereas enhanced DNMT3a expression resulted in decreased miR-200c expression $\left({ }^{*} \mathrm{P}<0.05,{ }^{* * *} \mathrm{P}<0.001\right)$.

To further explore the role of DNMT3a in regulating the expression of miR-200c, using siRNA against DNMT3a, we knocked down de novo DNMT3a expression in the AGS cells which had higher expression of DNMT3a when compared 


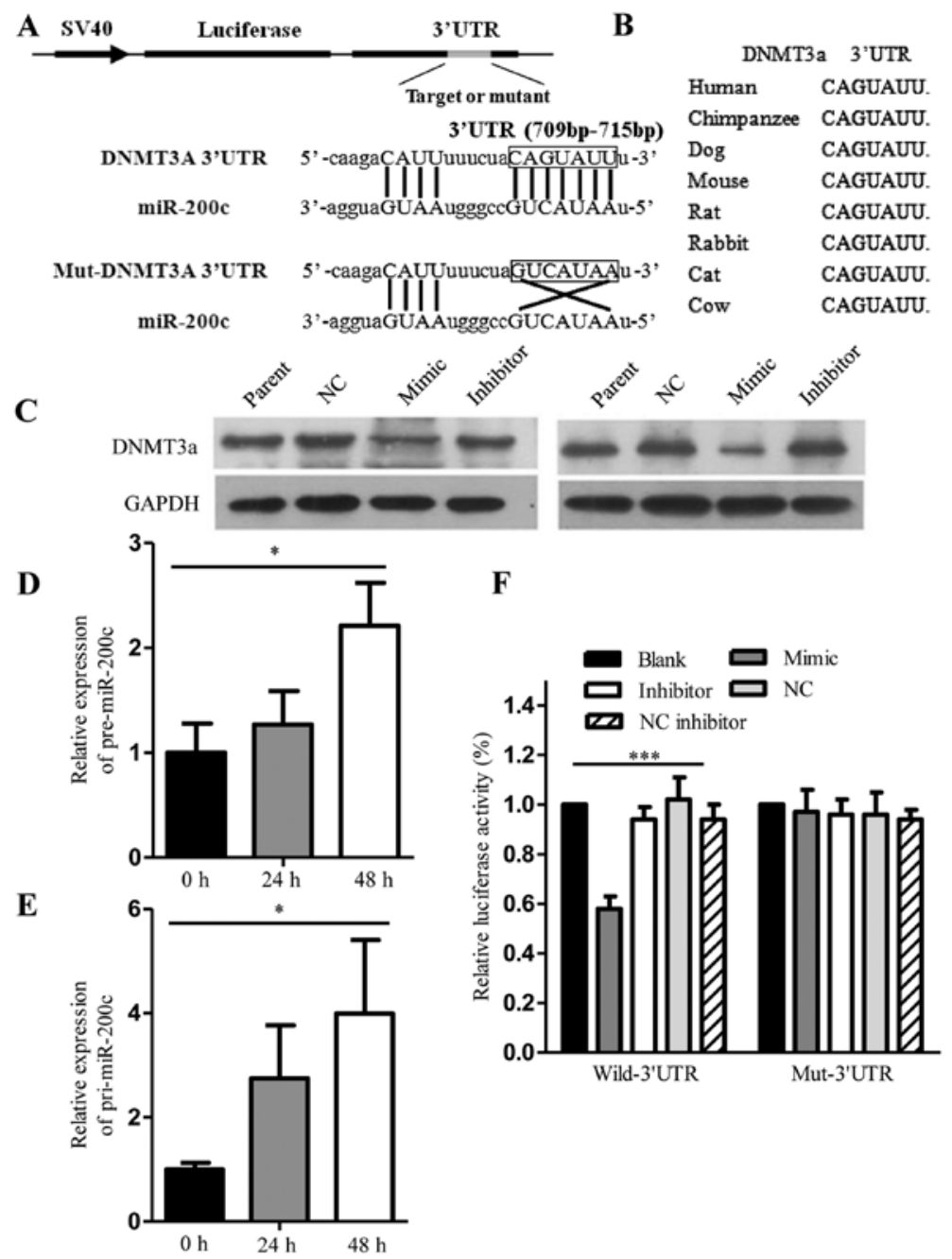

Figure 4. miR-200c suppresses DNMT3a expression and induces endogenous pre-miR-200c and pri-miR-200c reexpression. (A) Putative miR-200c binding sites within the human DNMT3a 3'UTR are shown. The position of the binding sites was numbered relative to the first nucleotide of the 3'UTR. Mutations were introduced into DNMT3a 3'UTR that matched the seed region of miR-200c. (B) Interspecies conservation of putative miR-200c binding sites within the DNMT3a 3'UTR are shown. (C) Transfection with miR-200c reduced DNMT3A protein expression, whereas transfection with miR-200c inhibitors increased DNMT3A protein expression in the SGC7901 (left) and AGS cells (right). (D and E) Relative expression of pre-miR-200c and pri-miR-200c demonstrated by qRT-PCR was increased in the AGS cells following 0,24 and $48 \mathrm{~h}$ transfection with $50 \mathrm{nM}$ miR-200c mimics. (F) Ectopic miR-200c expression inhibits wild-type but not mutant DNMT3A 3'UTR reporter activity in the SGC7901 cells. Cells were co-transfected with miR-200c mimics and with either wild-type or mutant DNMT3A 3'UTR reporter construct. Luciferase activity assay was performed at $48 \mathrm{~h}$ after transfection $\left({ }^{*} \mathrm{P}<0.05,{ }^{* * * *} \mathrm{P}<0.001\right)$.

with the other GC cell lines (Fig. 3A), and we enforced expression of DNMT3a in the MGC803 cells which had lower expression of DNMT3a when compared with the other GC cell lines (Fig. 3B). The results showed that DNMT3a knockdown abolished the hypermethylation of the miR-200c gene $(78.9 \%$ for untreated controls vs. $38.8 \%$ for DNMT3a siRNA; $\mathrm{P}<0.001$ ) (Fig. 3C and D), and induced upregulation of miR-200c expression $(\mathrm{P}<0.05)$ (Fig. 3E). Conversely, ectopic expression of DNMT3a increased methylation levels in the miR-200c promoter (78.3\% for untreated controls vs. $91.2 \%$ for DNMT3a overexpression; $\mathrm{P}<0.001$ ) (Fig. $3 \mathrm{C}$ and $\mathrm{D}$ ), and decreased miR-200c expression $(\mathrm{P}<0.05)$ (Fig. 3E). Together, these data demonstrate that DNMT3a regulates miR-200c expression via $\mathrm{CpG}$ island promoter hypermethylation.

miR-200c directly targets DNMT3a and induces endogenous pre-miR-200c and pri-miR-200c re-expression. As shown in Fig. 4A, one potential miR-200c targeting site was found in the 3'UTR of DNMT3a. Comparative sequence analysis showed that the target sequences were evolutionarily conserved in different species (Fig. 4B). Total RNA and protein was isolated from the SGC7901 and AGS cells collected $48 \mathrm{~h}$ after transfection. DNMT3a protein expression as demonstrated by western blot analysis was decreased in the SGC7901 and AGS cells transfected with miR-200c mimics (Fig. 4C). Additionally, pre-miR-200c and pri-miR-200c expression demonstrated by qRT-PCR was increased in the AGS cells transfected with the miR-200c mimics (Fig. 4D and E). By means of a luciferase reporter assay in the SGC7901 cells, we found that the luminescence intensity was significantly decreased in the DNMT3a 3'UTR reporter and miR-200c mimic-transfected cells than the others, suggesting that miR-200c has a target site in the 3'UTR of DNMT3a mRNA (Fig. 4F).

miR-200c mimics and DNMT3a siRNA suppress cell growth and invasion. The effects of miR-200c upregulation and DNMT3a downregulation on cell growth and invasion were determined via several assays in vitro. Transfection of 

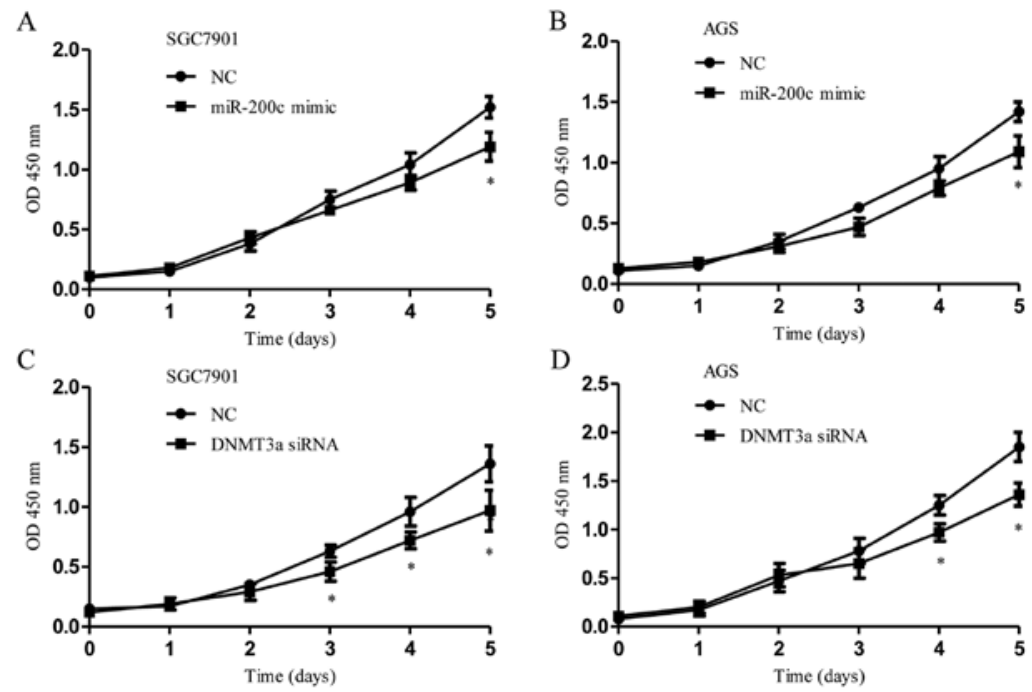

Figure 5. miR-200c mimics and DNMT3a siRNA suppress cell growth. (A and B) SGC7901 and AGS cells transfected with miR-200c mimics showed significantly reduced cellular proliferation compared with the negative control using Cell Counting Kit-8 assay. (C and D) SGC7901 and AGS cells transfected with DNMT3a siRNA also showed significantly reduced cellular proliferation compared with the negative control assaying ("P<0.05).

A

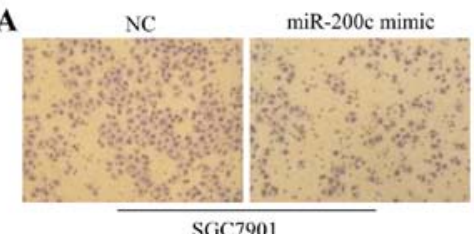

C

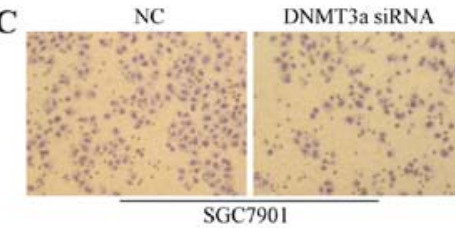

$\mathbf{E}$

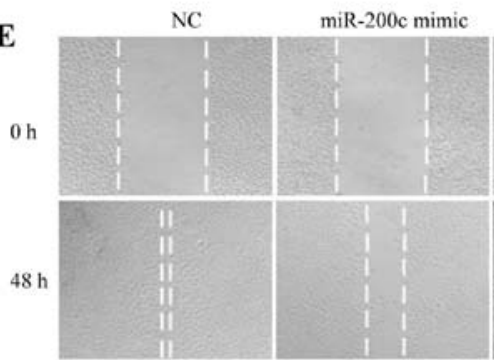

G

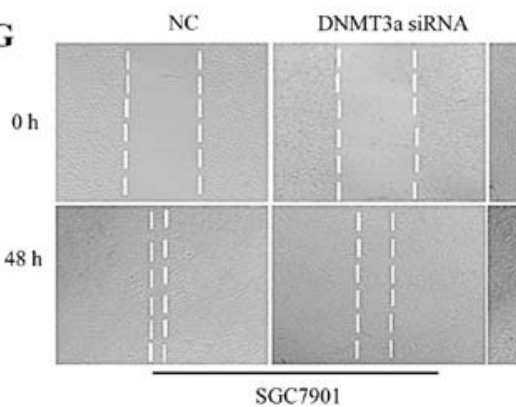

$\mathrm{NC}$

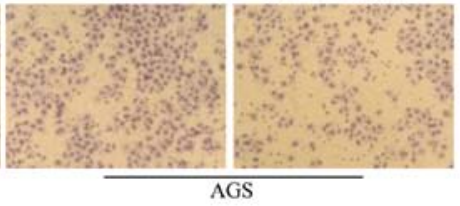

$\mathrm{NC}$

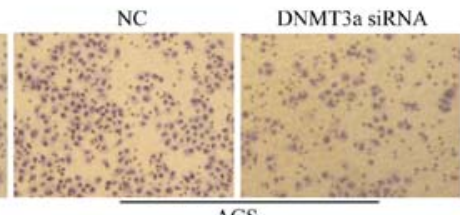

$\mathrm{NC}$

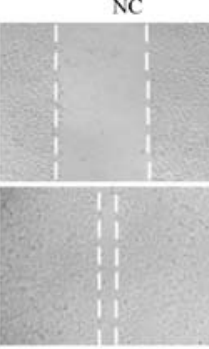

$\mathrm{NC}$

miR-200c mimic

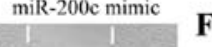

DNMT3a siRNA

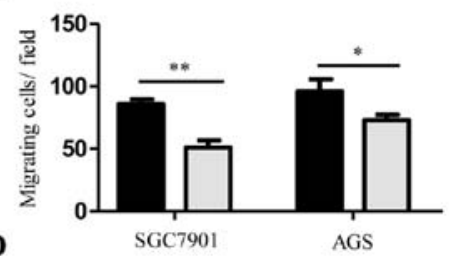

D
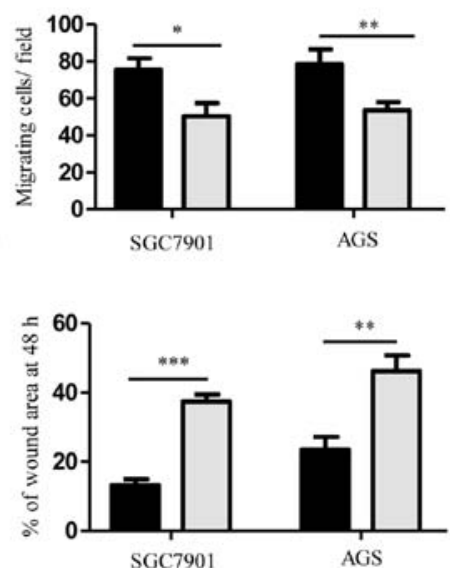

$\mathbf{H}$

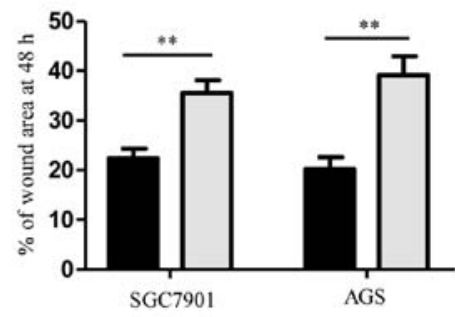

Figure 6. miR-200c mimics and DNMT3a siRNA suppress cell migration and invasion. (A) Representative images of cell invasion assays showed that miR200c mimics suppressed cell invasion in the SGC7901 and AGS cells. (B) Statistical analyses showed that miR-200c mimics suppressed cell invasion in the SGC7901 and AGS cells. (C) Representative images of cell invasion assays showed that DNMT3a siRNA suppressed cell invasion in the SGC7901 and AGS cells. (D) Statistical analyses showed that DNMT3a siRNA suppressed cell invasion in the SGC7901 and AGS cells. (E) Representative images of woundhealing assays showed that miR-200c mimics suppressed cell migration in the SGC7901 and AGS cells. (F) Statistical analyses showed that miR-200c mimics suppressed cell migration in the SGC7901 and AGS cells. (G) Representative images of wound-healing assays showed that DNMT3a siRNA suppressed cell migration in the SGC7901 and AGS cells. (H) Statistical analyses showed that DNMT3a siRNA suppressed cell migration in the SGC7901 and AGS cells $\left({ }^{*} \mathrm{P}<0.05,{ }^{* * *} \mathrm{P}<0.01,{ }^{* * * *} \mathrm{P}<0.001\right)$. 
miR-200c mimics or DNMT3a siRNA significantly reduced cell proliferation in the SGC7901 and AGS cells compared to the proliferation noted in the control transfections (Fig. 5A-D). Upregulation of miR-200c or knockdown of DNMT3a in the SGC7901 and AGS cells significantly inhibited cell invasion (Fig. 6A-D). The effects of miR-200c on cell migration were determined by wound-healing assays. Both SGC7901 and AGS cells treated with miR-200c mimics or DNMT3a siRNA were distinctively less migratory than that noted in the negative control cells (Fig. 6E-H). These results indicate that upregulation of miR-200c or knockdown of DNMT3a suppressed cell growth, migration and invasion of GC cells in vitro.

\section{Discussion}

Gastric carcinogenesis is a multistep process characterized by accumulation of molecular alterations (18). A complicated reciprocal repression mechanism between miRNAs and epigenetic pathways appears to form an epigenetic-miRNA network contributing to gastric carcinogenesis (19). In the present study, we found that miR-200c was downregulated in $\mathrm{GC}$ and was associated with the histologic type in GC. Dysregulation of miR-200c in GC has also been observed in other studies $(20,21)$, but little is known concerning its expression in diffuse-type GC. This is the first study to show that miR-200c is downregulated in diffuse-type GC.

It is well recognized that miR-200c regulates the epithelial-mesenchymal transition (EMT) and cancer cell invasion $(22,23)$. Ectopic expression of miR-200 hinders EMT by suppressing ZEB1 and ZEB2 expression and enhancing E-cadherin expression (24,25). Notably, EMT is an important process to form diffuse histology and initiate metastasis by enhancing the motility of cancer cells (18). Therefore, reduced expression of miR-200c may participate in the genesis of diffuse histology of GC by reducing E-cadherin expression through ZEB1 and ZEB2.

We next demonstrated that miR-200c expression is regulated by DNA methylation. It is widely accepted that miRNAs undergo the same regulatory mechanisms of the protein coding gene, including methylation regulation. Generally, DNA methylation is accomplished by three enzymes: DNMT1, DNMT3a and DNMT3b. DNMT1 is responsible for maintaining preexisting methylation patterns during DNA replication, whereas DNMT3a and DNMT3b are considered to be de novo DNA methyltransferase (26). In breast, miR-200c was repressed by DNA methylation, whereas the miR-200b-200a-429 cluster was silenced primarily through polycomb group-mediated histone modifications (27), suggesting that epigenetic control of miRNAs may be miRNA-specific and epigenetic effector-specific. The increased expression of DNMT3a may play an important role in the malignant progression of cancer, leading to aberrant methylation in many important tumor suppressor genes (28). More recently, Cao et al (29) reported that the expression of DNMT3a is an independent poor prognostic indicator in GC. Yang et al (30) reported that overexpression of DNMT3a in GC tissues was observed in $70.4 \%$ of 54 cases, and DNMT3a was associated with TNM stage and lymph node metastasis. Aberrant expression of DNMT3a may cause upregulation of global DNA methylation, decreasing expression of tumor suppressor genes that may contribute to aggressive poorly differentiated cancer. However, the mechanisms underlying aberrant DNMT expression have not been fully elucidated.

Our results showed that miR-200c can target 3'UTR of DNMT3a directly; in contrast, DNMT3a can establish and maintain miR-200c methylation to suppress its expression. Thus, DNMT3a and miR-200c form a negative feedback loop in GC cells, and this may plays a key role in gastric carcinogenesis. Most investigations which focused on the mutual regulation between DNA methylation and miRNAs were frequently confirmed in fibrotic disease and cancer research. For example, a mutual regulation of miRNAs and DNA methylation may be involved in the pathogenesis of lung fibrosis (31). A regulatory circuit of miR-148a/152 and DNMT1 was found to modulate breast cancer cell transformation and tumor angiogenesis through IGF-IR and IRS1 (9). Another study also demonstrated that miR-152 and DNMT1 interacted with each other via a feedback loop involved in NiS-induced malignant transformation (32). In esophageal squamous cell carcinoma, the DNMT1-miRNA-126 epigenetic circuit was contributed to cancer growth via ADAM9/EGFR/AKT signaling (33). Thus, epigenetic-miRNA loops are widely present in various diseases including cancers. Given that both miRNA and epigenetic modulations strongly regulate the expression of various disease-associated genes (34), controlling the dynamic balance of epigenetic-miRNA loops may be of great clinical significance.

Regulation of DNMT3a by miR-200c has also been described in GC by Tang et al (20), but the mutual regulation between DNMT3a and miR-200c has not been confirmed in previous studies. The results indicate that DNMT3a is a crucial regulator, as its aberrant expression in cancer may start a self-enhancing feed forward loop by downregulating its own inhibitor miR-200c. It has been reported that a reciprocal repression between ZEB1 and the miR-200 family promotes EMT and invasion in cancer cells (35), thus, it is plausible that aberrant expression of DNMT3a may interact with the reciprocal repression between ZEB1 and miR-200c, thereby inducing downregulation of miR-200c and upregulation of ZEB1, subsequently promoting EMT and invasion in cancer cells. Indeed, in vitro invasion and migration assays implicate that the loop between miR-200c and DNMT3a is involved in promoting EMT and metastasis of GC cells. Future experiments are needed to determine whether both DNMT3a and ZEB1 act separately or synergistically as a complex in this setting.

In conclusion, miR-200c directly suppressed the expression levels of DNMT3a. In contrast, overexpression of DNMT3a was responsible for the promoter hypermethylation of miR-200c. Downregulated expression of miR-200c further increased DNMT3a expression by less targeting DNMT3a 3'UTR. A novel miR-200c-DNMT3a regulatory circuit may exist in GC. The discovery of this miRNA-epigenetic regulatory circuit would be highly beneficial for deepening our understanding of gastric carcinogenesis.

\section{Acknowledgements}

The present study was supported by the National Natural Science Foundation of China (no. 81372633), the National 
Natural Science Foundation of China (no. 81502040), the Projects of Medical and Health Technology Program in Guangzhou City (no. 20161A010009), and the Key Programs of the Health Bureau of Guangzhou City (20121A021004).

\section{References}

1. Torre LA, Bray F, Siegel RL, Ferlay J, Lortet-Tieulent J and Jemal A: Global cancer statistics, 2012. CA Cancer J Clin 65 : 87-108, 2015

2. Lin S and Gregory RI: MicroRNA biogenesis pathways in cancer. Nat Rev Cancer 15: 321-333, 2015.

3. Ueda T, Volinia S, Okumura H, Shimizu M, Taccioli C, Rossi S, Alder H, Liu CG, Oue N, Yasui W, et al: Relation between microRNA expression and progression and prognosis of gastric cancer: A microRNA expression analysis. Lancet Oncol 11: 136-146, 2010.

4. Song S and Ajani JA: The role of microRNAs in cancers of the upper gastrointestinal tract. Nat Rev Gastroenterol Hepatol 10: $109-118,2013$.

5. Ando T, Yoshida T, Enomoto S, Asada K, Tatematsu M, Ichinose M, Sugiyama T and Ushijima T: DNA methylation of microRNA genes in gastric mucosae of gastric cancer patients: Its possible involvement in the formation of epigenetic field defect. Int J Cancer 124: 2367-2374, 2009.

6. Kiga K, Mimuro H, Suzuki M, Shinozaki-Ushiku A, Kobayashi T, Sanada T, Kim M, Ogawa M, Iwasaki YW, Kayo H, et al: Epigenetic silencing of miR-210 increases the proliferation of gastric epithelium during chronic Helicobacter pylori infection. Nat Commun 5: 4497, 2014.

7. Li Z, Lei H, Luo M, Wang Y, Dong L, Ma Y, Liu C, Song W, Wang F, Zhang J, et al: DNA methylation downregulated mir-10b acts as a tumor suppressor in gastric cancer. Gastric Cancer 18: 43-54, 2015.

8. Morita S, Horii T, Kimura M, Ochiya T, Tajima S and Hatada I: miR-29 represses the activities of DNA methyltransferases and DNA demethylases. Int J Mol Sci 14: 14647-14658, 2013.

9. Xu Q, Jiang Y, Yin Y, Li Q, He J, Jing Y, Qi YT, Xu Q, Li W, Lu B, et al: A regulatory circuit of miR-148a/152 and DNMT1 in modulating cell transformation and tumor angiogenesis through IGF-IR and IRS1. J Mol Cell Biol 5: 3-13, 2012.

10. Xiang Y, Ma N, Wang D, Zhang Y, Zhou J, Wu G, Zhao R, Huang H, Wang X, Qiao Y, et al: MiR-152 and miR-185 co-contribute to ovarian cancer cells cisplatin sensitivity by targeting DNMT1 directly: A novel epigenetic therapy independent of decitabine. Oncogene 33: 378-386, 2014.

11. Huang J, Wang Y, Guo Y and Sun S: Down-regulated microRNA-152 induces aberrant DNA methylation in hepatitis B virus-related hepatocellular carcinoma by targeting DNA methyltransferase 1. Hepatology 52: 60-70, 2010.

12. Song F, Yang D, Liu B, Guo Y, Zheng H, Li L, Wang T, Yu J, Zhao Y, Niu R, et al: Integrated microRNA network analyses identify a poor-prognosis subtype of gastric cancer characterized by the miR-200 family. Clin Cancer Res 20: 878-889, 2014.

13. Kurashige J, Mima K, Sawada G, Takahashi Y, Eguchi H, Sugimachi K, Mori M, Yanagihara K, Yashiro M, Hirakawa K, et al: Epigenetic modulation and repression of miR-200b by cancer-associated fibroblasts contribute to cancer invasion and peritoneal dissemination in gastric cancer. Carcinogenesis 36: 133-141, 2015.

14. Ning X, Shi Z, Liu X, Zhang A, Han L, Jiang K, Kang C and Zhang Q: DNMT1 and EZH2 mediated methylation silences the microRNA-200b/a/429 gene and promotes tumor progression. Cancer Lett 359: 198-205, 2015.

15. Davalos V, Moutinho C, Villanueva A, Boque R, Silva P, Carneiro F and Esteller M: Dynamic epigenetic regulation of the microRNA-200 family mediates epithelial and mesenchymal transitions in human tumorigenesis. Oncogene 31: 2062-2074, 2012.

16. Hur K, Toiyama Y, Takahashi M, Balaguer F, Nagasaka T, Koike J, Hemmi H, Koi M, Boland CR and Goel A: MicroRNA-200c modulates epithelial-to-mesenchymal transition (EMT) in human colorectal cancer metastasis. Gut 62: 1315-1326, 2013.
17. Neves R, Scheel C, Weinhold S, Honisch E, Iwaniuk KM, Trompeter HI, Niederacher D, Wernet P, Santourlidis S and Uhrberg M: Role of DNA methylation in miR-200c/141 cluster silencing in invasive breast cancer cells. BMC Res Notes 3: 219, 2010.

18. Yasui W, Sentani K, Sakamoto N, Anami K, Naito Y and Oue N: Molecular pathology of gastric cancer: Research and practice. Pathol Res Pract 207: 608-612, 2011.

19. Gigek CO, Chen ES, Calcagno DQ, Wisnieski F, Burbano RR and Smith MA: Epigenetic mechanisms in gastric cancer. Epigenomics 4: 279-294, 2012.

20. Tang H, Deng M, Tang Y, Xie X, Guo J, Kong Y, Ye F, Su Q and Xie X: miR-200b and miR-200c as prognostic factors and mediators of gastric cancer cell progression. Clin Cancer Res 19: 5602-5612, 2013

21. Zhou X, Wang Y, Shan B, Han J, Zhu H, Lv Y, Fan X, Sang M, Liu XD and Liu W: The downregulation of miR-200c/141 promotes ZEB1/2 expression and gastric cancer progression. Med Oncol 32: 428, 2015.

22. Mongroo PS and Rustgi AK: The role of the miR-200 family in epithelial-mesenchymal transition. Cancer Biol Ther 10: 219-222, 2010.

23. Feng X, Wang Z, Fillmore R and Xi Y: MiR-200, a new star miRNA in human cancer. Cancer Lett 344: 166-173, 2014.

24. Paterson EL, Kazenwadel J, Bert AG, Khew-Goodall Y, Ruszkiewicz A and Goodall GJ: Down-regulation of the miRNA-200 family at the invasive front of colorectal cancers with degraded basement membrane indicates EMT is involved in cancer progression. Neoplasia 15: 180-191, 2013.

25. Ahn SM, Cha JY, Kim J, Kim D, Trang HT, Kim YM, Cho YH, Park D and Hong S: Smad3 regulates E-cadherin via miRNA-200 pathway. Oncogene 31: 3051-3059, 2012.

26. Jin B and Robertson KD: DNA methyltransferases, DNA damage repair, and cancer. Adv Exp Med Biol 754: 3-29, 2013.

27. Lim YY, Wright JA, Attema JL, Gregory PA, Bert AG, Smith E, Thomas D, Lopez AF, Drew PA, Khew-Goodall Y, et al: Epigenetic modulation of the miR-200 family is associated with transition to a breast cancer stem-cell-like state. J Cell Sci 126: 2256-2266, 2013

28. Daniel FI, Cherubini K, Yurgel LS, de Figueiredo MA and Salum FG: The role of epigenetic transcription repression and DNA methyltransferases in cancer. Cancer 117: 677-687, 2011.

29. Cao XY, Ma HX, Shang YH, Jin MS, Kong F, Jia ZF, Cao DH, Wang YP, Suo J and Jiang J. DNA methyltransferase3a expression is an independent poor prognostic indicator in gastric cancer. World J Gastroenterol 20: 8201-8208, 2014.

30. Yang J, Wei X, Wu Q, Xu Z, Gu D, Jin Y, Shen Y, Huang H, Fan $\mathrm{H}$ and Chen $\mathrm{J}$ : Clinical significance of the expression of DNA methyltransferase proteins in gastric cancer. Mol Med Rep 4: 1139-1143, 2011.

31. Dakhlallah D, Batte K, Wang Y, Cantemir-Stone CZ, Yan P, Nuovo G, Mikhail A, Hitchcock CL, Wright VP, Nana-Sinkam SP, et al: Epigenetic regulation of miR-17 92 contributes to the pathogenesis of pulmonary fibrosis. Am J Respir Crit Care Med 187: 397-405, 2013.

32. Ji W, Yang L, Yuan J, Yang L, Zhang M, Qi D, Duan X, Xuan A, Zhang W, Lu J, et al: MicroRNA-152 targets DNA methyltransferase 1 in NiS-transformed cells via a feedback mechanism. Carcinogenesis 34: 446-453, 2013.

33. Liu R, Gu J, Jiang P, Zheng Y, Liu X, Jiang X, Huang E, Xiong S, $\mathrm{Xu}$ F, Liu G, et al: DNMT1-microRNA126 epigenetic circuit contributes to esophageal squamous cell carcinoma growth via ADAM9-EGFR-AKT signaling. Clin Cancer Res 21: 854-863, 2015.

34. Jones PA and Baylin SB: The fundamental role of epigenetic events in cancer. Nat Rev Genet 3: 415-428, 2002.

35. Burk U, Schubert J, Wellner U, Schmalhofer O, Vincan E, Spaderna S and Brabletz T: A reciprocal repression between ZEB1 and members of the miR-200 family promotes EMT and invasion in cancer cells. EMBO Rep 9: 582-589, 2008. 